TAIWANESE JOURNAL OF MATHEMATICS

Vol. 19, No. 1, pp. 65-75, February 2015

DOI: $10.11650 /$ tjm.19.2015.4411

This paper is available online at http://journal.taiwanmathsoc.org.tw

\title{
ON THE NUMBER OF LAPLACIAN EIGENVALUES OF TREES SMALLER THAN TWO
}

\author{
Lingling Zhou, Bo Zhou* and Zhibin Du
}

\begin{abstract}
Let $m_{T}[0,2)$ be the number of Laplacian eigenvalues of a tree $T$ in $[0,2)$, multiplicities included. We give best possible upper bounds for $m_{T}[0,2)$ using the parameters such as the number of pendant vertices, diameter, matching number, and domination number, and characterize the trees $T$ of order $n$ with $m_{T}[0,2)=n-1, n-2$, and $\left\lceil\frac{n}{2}\right\rceil$, respectively, and in particular, show that $m_{T}[0,2)=\left\lceil\frac{n}{2}\right\rceil$ if and only if the matching number of $T$ is $\left\lfloor\frac{n}{2}\right\rfloor$.
\end{abstract}

\section{INTRODUCTION}

We consider simple graphs. Let $G$ be a graph with vertex set $V(G)$. For $v \in$ $V(G)$, let $d_{G}(v)$ be the degree of $v$ in $G$. The Laplacian matrix of $G$ is defined as $L(G)=D(G)-A(G)$, where $D(G)$ is the degree diagonal matrix of $G$, and $A(G)$ is the adjacency matrix of $G$. The Laplacian eigenvalues of $G$ are the eigenvalues of $L(G)$. Since $L(G)$ is a positive semi-definite matrix, the Laplacian eigenvalues of $G$ are nonnegative real numbers. Let $\mu_{1}(G) \leq \mu_{2}(G) \leq \cdots \leq \mu_{n}(G)$ be the Laplacian eigenvalues of $G$, arranged in nondecreasing order, where $n=|V(G)|$. Since each row sum of $L(G)$ is zero, $\mu_{1}(G)=0$. Recall that $\mu_{n}(G) \leq n$ (see $[1,5]$ ). Thus all Laplacian eigenvalues of $G$ belong to $[0, n]$. For a survey on Laplacian eigenvalues, see [11].

For a graph $G$ on $n$ vertices and an interval $I \subseteq[0, n]$, let $m_{G} I$ be the number of Laplacian eigenvalues of $G$, multiplicities included, that belong to $I$.

Grone and Merris [5] showed that for a graph with at least one edge, its largest Laplacian eigenvalue is at least the maximum degree plus one. Thus for a tree $T$ on $n \geq 2$ vertices, $m_{T}[0,2) \leq n-1$.

Received February 8, 2014, accepted April 28, 2014.

Communicated by Gerard Jennhwa Chang.

2010 Mathematics Subject Classification: 05C50, 05C35.

Key words and phrases: Laplacian eigenvalues, Trees, Pendant vertex, Diameter, Matching number, Domination number.

*Corresponding author. 
A vertex of a graph $G$ is a pendant vertex if $d_{G}(v)=1$. A vertex of $G$ is a quasi-pendant vertex if it is adjacent to a pendant vertex.

For a graph $G$ on $n$ vertices with $p$ pendant vertices, $q$ quasi-pendant vertices, and diameter $d$, Grone et al. [6] showed that

$$
\begin{aligned}
m_{G}[0,1], m_{G}[1, n] & \geq p, \\
m_{G}[0,1), m_{G}(1, n] & \geq q, \\
m_{G}(2, n] & \geq\left\lfloor\frac{d}{2}\right\rfloor,
\end{aligned}
$$

and Merris [10] showed that if $n>2 q$, then

$$
m_{G}(2, n] \geq q .
$$

Braga et al. [3] showed that for a tree $T$ on $n \geq 2$ vertices,

$$
m_{T}[0,2) \geq\left\lceil\frac{n}{2}\right\rceil \text {. }
$$

More results along this line may be found in $[3,7,8]$.

In this paper, we give best possible upper bounds for $m_{T}[0,2)$ using the parameters of a tree $T$ such as the number of pendant vertices, diameter, matching number, and domination number, provide a simple different proof for the lower bound in [3] mentioned above, characterize the trees $T$ of order $n$ with $m_{T}[0,2)=n-1, n-2$, and $\left\lceil\frac{n}{2}\right\rceil$, respectively, and in particular, show that $m_{T}[0,2)=\left\lceil\frac{n}{2}\right\rceil$ if and only if the matching number of $T$ is $\left\lfloor\frac{n}{2}\right\rfloor$ (in Theorem 4.2).

\section{Preliminaries}

An algorithm for computing the number of Laplacian eigenvalues of a tree in an interval was proposed in [3] based on the algorithm for computing the number of adjacency eigenvalues of a tree in an interval [9]. For a tree $T$ on $n$ vertices, choose any vertex as the root of $T$, and label the vertices of $T$ as $v_{1}, v_{2}, \ldots, v_{n}$ such that if $v_{i}$ is a child of $v_{k}$, then $k>i$. The algorithm for computing $m_{T}[0,2)$ of a tree $T$ is given as follows:

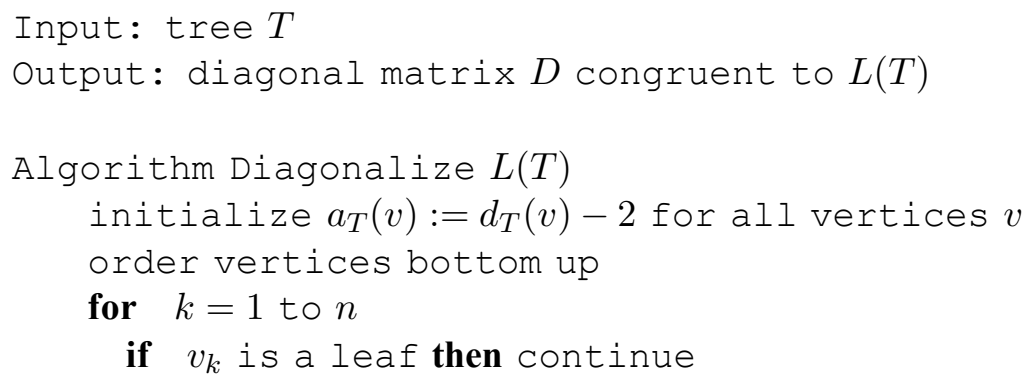




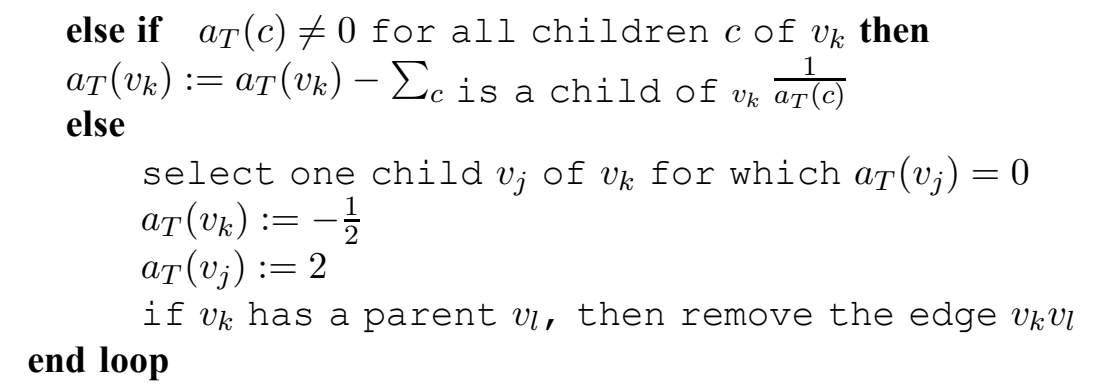

For a tree $T$ with vertices $v_{1}, v_{2}, \ldots, v_{n}$ labelled as above, the weight of $v_{i}$ in $T$ is the $i$-th diagonal entry $a_{T}\left(v_{i}\right)$ of the diagonal matrix $D$ obtained under the above algorithm, where $1 \leq i \leq n$. If $a_{T}\left(v_{i}\right)<0$, we say $v_{i}$ has a negative weight in $T$.

Lemma 2.1. [3]. Suppose that $T$ is a tree. Then $m_{T}[0,2)$ is equal to the number of vertices with negative weights in $T$.

A double broom is a tree obtained by attaching some pendant vertices to the two end vertices of a path on at least two vertices. In particular, a star is also regarded as a double broom.

Lemma 2.2. Let $T$ be an $n$-vertex double broom with diameter $d$, where $1 \leq d \leq$ $n-1$. Then $m_{T}[0,2)=\left\lfloor\frac{2 n-d}{2}\right\rfloor$.

Proof. Choosing a quasi-pendant vertex of $T$ as the root of $T$. Then the result follows from Lemma 2.1 easily.

Lemma 2.3. Let $T$ be a tree with $v \in V(T)$, and $T^{\prime}$ be the tree obtained from $T$ by attaching $a$ path on two vertices to $v$. Then $m_{T^{\prime}}[0,2)=m_{T}[0,2)+1$.

Proof. In both $T$ and $T^{\prime}$, we choose $v$ as the root. Note that the two vertices in $T^{\prime}$ not in $T$ have weights 1 and -1 , and $a_{T}(x)=a_{T^{\prime}}(x)$ for $x \in V(T)$. Then the result follows from Lemma 2.1 clearly.

Lemma 2.4. Let $T$ be a tree with $v \in V(T)$, and $T^{*}$ be the tree obtained from $T$ by attaching two pendant vertices to $v$. Then $m_{T^{*}}[0,2) \geq m_{T}[0,2)+1$.

Proof. Let us choose $v$ as the root of both $T$ and $T^{*}$. Clearly, $a_{T}(x)=a_{T^{*}}(x)$ for $x \in V(T) \backslash\{v\}$. Denote by $s$ the number of vertices in $T$ different from $v$ with negative weights. Note that each pendant vertex in $T^{*}$ has weight -1 . By Lemma 2.1, $m_{T^{*}}[0,2) \geq s+2=(s+1)+1 \geq m_{T}[0,2)+1$.

Lemma 2.5. [6] . Let $G$ be an n-vertex graph and $G^{\prime}$ a graph obtained from $G$ by deleting an edge. Then

$$
0=\mu_{1}\left(G^{\prime}\right)=\mu_{1}(G) \leq \mu_{2}\left(G^{\prime}\right) \leq \mu_{2}(G) \leq \cdots \leq \mu_{n}\left(G^{\prime}\right) \leq \mu_{n}(G) .
$$


For a vertex $v$ of a graph $G, G-v$ denotes the graph resulting from $G$ by deleting $v$ (and its incident edges). For an edge $u v$ of a graph $G$ (the complement of $G$, respectively), $G-u v(G+u v$, respectively) denotes the graph resulting from $G$ by deleting (adding, respectively) $u v$.

\section{UPPER BOUNDS FOR $m_{T}[0,2)$}

For a tree $T$, if $v$ is a vertex of $T$ with exactly $d_{T}(v)-1 \geq 1$ pendant neighbors, then the subgraph induced by $v$ and its $d_{T}(v)-1$ pendant neighbors is said to be a pendant star of $T$ at $v$. If $T$ is not a star, then $T$ has some pendant stars.

Lemma 3.1. Suppose that $T$ is a tree with a pendant star at $v$, say $T_{1}$. If we choose a vertex of $T$ outside $T_{1}$ as the root of $T$, then $a_{T}(v)>0$.

Proof. Clearly, $a_{T}(u)=-1$ for any pendant neighbor $u$ of $v$ in $T$. Thus

$$
a_{T}(v)=d_{T}(v)-2-\left(d_{T}(v)-1\right) \frac{1}{a_{T}(u)}=2 d_{T}(v)-3>0,
$$

as desired.

Lemma 3.2. Let $T$ be a tree, and $T_{1}$ be the tree obtained from $T$ by deleting $a$ pendant vertex. Then $m_{T}[0,2)=m_{T_{1}}[0,2)$ or $m_{T_{1}}[0,2)+1$.

Proof. Let $v$ be a pendant vertex of $T$, being adjacent to $u$. By Lemma 2.5, $\mu_{i}(T) \leq \mu_{i+1}(T-u v) \leq \mu_{i+1}(T)$ for $1 \leq i \leq n-1$. Obviously, $T-u v$ consists of $T_{1}$ and an isolated vertex $v$. Thus $\mu_{i+1}(T-u v)=\mu_{i}\left(T_{1}\right)$ for $1 \leq i \leq n-1$. It follows that $\mu_{i}(T) \leq \mu_{i}\left(T_{1}\right) \leq \mu_{i+1}(T)$ for $1 \leq i \leq n-1$. From $\mu_{i}(T) \leq \mu_{i}\left(T_{1}\right)$, we have $m_{T}[0,2) \geq m_{T_{1}}[0,2)$, and from $\mu_{i}\left(T_{1}\right) \leq \mu_{i+1}(T)$, we have $m_{T_{1}}[0,2) \geq m_{T}[0,2)-1$. Thus we have the desired result.

Theorem 3.1. Let $T$ be an $n$-vertex tree with p pendant vertices, where $2 \leq p \leq$ $n-1$. Then $m_{T}[0,2) \leq\left\lfloor\frac{n+p-1}{2}\right\rfloor$.

Proof. We prove the result by induction on $n$.

If $n=3$, then $T$ is a star with $p=2$, and by Lemma $2.2, m_{T}[0,2)=2 \leq\left\lfloor\frac{n+p-1}{2}\right\rfloor$.

Suppose that the result holds for all trees on less than $n \geq 4$ vertices with any possible number of pendant vertices. Let $T$ be an $n$-vertex tree with $p$ pendant vertices. Let $v$ be an end vertex of a diametrical path of $T$, and $u$ be the (unique) neighbor of $v$ (on that diametrical path).

Suppose first that $u$ is of degree two. Note that $T-v-u$ has at most $p$ pendant vertices. Applying the induction hypothesis to $T-v-u$, we have $m_{T-v-u}[0,2) \leq$ $\left\lfloor\frac{(n-2)+p-1}{2}\right\rfloor$. Then by Lemma 2.3, we have 


$$
m_{T}[0,2)=m_{T-v-u}[0,2)+1 \leq\left\lfloor\frac{(n-2)+p-1}{2}\right\rfloor+1=\left\lfloor\frac{n+p-1}{2}\right\rfloor .
$$

Now suppose that $u$ is of degree at least three. Note that $T-v$ has $p-1$ pendant vertices. Applying the induction hypothesis to $T-v$, we have $m_{T-v}[0,2) \leq\left\lfloor\frac{(n-1)+(p-1)-1}{2}\right\rfloor$. Then by Lemma 3.2, we have

$$
m_{T}[0,2) \leq m_{T-v}[0,2)+1 \leq\left\lfloor\frac{(n-1)+(p-1)-1}{2}\right\rfloor+1=\left\lfloor\frac{n+p-1}{2}\right\rfloor .
$$

The result follows.

Corollary 3.1. Let $T$ be an $n$-vertex tree with diameter $d$, where $2 \leq d \leq n-1$. Then $m_{T}[0,2) \leq\left\lfloor\frac{2 n-d}{2}\right\rfloor$.

Proof. Denote by $p$ the number of pendant vertices in $T$. Clearly, $p \leq n-d+1$. Then the result follows from Theorem 3.1 easily.

The upper bounds in Theorem 3.1 and Corollary 3.1 are both tight since they are attained when $T$ is an $n$-vertex double broom.

A matching of a graph is an edge subset in which no pair shares a common vertex. The matching number $\beta(G)$ of a graph $G$ is the maximum cardinality of a matching of $G$.

Theorem 3.2. Let $T$ be an n-vertex tree with matching number $\beta$, where $1 \leq \beta \leq$ $\left\lfloor\frac{n}{2}\right\rfloor$. Then $m_{T}[0,2) \leq n-\beta$.

Proof. We prove the result by induction on $n$.

The case $n=3$ follows obviously from Lemma 2.2.

Suppose that the result holds for all trees on less than $n \geq 4$ vertices with any possible matching number. Let $T$ be an $n$-vertex tree with matching number $\beta$. Let $v$ be an end vertex of a diametrical path of $T$, and $u$ be the (unique) neighbor of $v$ (on that diametrical path).

Suppose first that $u$ is of degree two. Note that $T-v-u$ has matching number $\beta-1$. Applying the induction hypothesis to $T-v-u$, we have $m_{T-v-u}[0,2) \leq$ $(n-2)-(\beta-1)=n-\beta-1$. Now it follows from Lemma 2.3 that

$$
m_{T}[0,2)=m_{T-v-u}[0,2)+1 \leq(n-\beta-1)+1=n-\beta .
$$

Now suppose that $u$ is of degree at least three. Note that $T-v$ has matching number $\beta$. Applying the induction hypothesis to $T-v$, we have $m_{T-v}[0,2) \leq n-1-\beta$. Now it follows from Lemma 3.2 that

$$
m_{T}[0,2) \leq m_{T-v}[0,2)+1 \leq(n-1-\beta)+1=n-\beta .
$$


The result follows.

A dominating set of a graph is a vertex subset whose closed neighborhood contains all vertices of the graph. The domination number of a graph $G$ is the minimum cardinality of a dominating set of $G$.

A covering of a graph $G$ is a vertex subset $K$ such that every edge of $G$ has at least one end vertex in $K$.

Corollary 3.2. Let $T$ be an n-vertex tree with domination number $\gamma$, where $1 \leq$ $\gamma \leq\lfloor n / 2\rfloor$. Then $m_{T}[0,2) \leq n-\gamma$.

Proof. Denote by $\beta$ the matching number of $T$. By König's theorem [2], $\beta$ is equal to the minimum cardinality of a covering of $G$. Note that a covering of $T$ is also a dominating set of $T$. Thus $\beta \geq \gamma$. Then the result follows from Theorem 3.2 easily.

The upper bounds in Theorem 3.2 and Corollary 3.2 are both tight since they are attained when $T$ is an $n$-vertex tree obtained by attaching some paths on two vertices to the central vertex of a star.

Recall that $m_{T}[0,2) \leq n-1$ for any tree $T$ on $n \geq 2$ vertices [5], (which also follows from Theorem 3.2). Let $\mathcal{T}_{n}^{1}$ be the set of $n$-vertex trees (double brooms) with diameter three, where $n \geq 4$. Let $\mathcal{T}_{n}^{2}$ be the set of $n$-vertex double brooms with diameter four, where $n \geq 5$.

Theorem 3.3. Let $T$ be a tree on $n$ vertices.

(i) $m_{T}[0,2)=n-1$ for $n \geq 2$ if and only if $T \cong S_{n}$.

(ii) $m_{T}[0,2)=n-2$ for $n \geq 4$ if and only if $T \in \mathcal{T}_{n}^{1} \cup \mathcal{T}_{n}^{2}$.

Proof. By Lemma 2.2, $m_{T}[0,2)=n-1$ if $T \cong S_{n}$, and $m_{T}[0,2)=n-2$ if $T \in \mathcal{T}_{n}^{1} \cup \mathcal{T}_{n}^{2}$.

Suppose in the following that $T \notin\left\{S_{n}\right\} \cup \mathcal{T}_{n}^{1} \cup \mathcal{T}_{n}^{2}$. Then $n \geq 6$. Let $P=$ $v_{0} v_{1} \ldots v_{d}$ be a diametrical path of $T$. Obviously, $d \geq 4$. Let $T_{1}$ be the pendant star of $T$ at $v_{1}$, and $T_{2}$ be the pendant star of $T$ at $v_{d-1}$.

If $T_{1}$ and $T_{2}$ are the only two vertex-disjoint pendant stars in $T$, then $T$ is a double broom with $d \geq 5$, and thus by Lemma $2.2, m_{T}[0,2) \leq n-3$.

Suppose that there are at least three vertex-disjoint pendant stars in $T$. Let $T_{3}$ be a pendant star in $T$ different from $T_{1}$ and $T_{2}$.

If $V(T)=V\left(T_{1}\right) \cup V\left(T_{2}\right) \cup V\left(T_{3}\right)$, then $T$ is the tree obtained by attaching at least one pendant vertex to each vertex of $P_{3}$, and by choosing $v_{2}$ as the root of $T$ and applying Lemma 2.1, we have $m_{T}[0,2)=n-3$.

Suppose that $V(T) \supset V\left(T_{1}\right) \cup V\left(T_{2}\right) \cup V\left(T_{3}\right)$. Let $u$ be a vertex in $T$ outside $T_{1}, T_{2}, T_{3}$. Choosing $u$ as the root of $T$, and by Lemma 3.1, each of $T_{1}, T_{2}, T_{3}$ has one vertex which is not of negative weight. Thus, by Lemma 2.1, we have $m_{T}[0,2) \leq n-3$.

Now the result follows easily. 


\section{A LOWER BOUND FOR $m_{T}[0,2)$}

For a tree $T$, if $u$ is a pendant vertex of $T$ being adjacent to a vertex $v$ of degree two, then the subgraph of $T$ induced by $u$ and $v$ is said to be a pendant $P_{2}$ of $T$. For a tree on at least three vertices, if there is no pendant $P_{2}$, then there are two pendant vertices sharing a common neighbor.

Deleting a pendant $P_{2}$ of a tree $T$ is said to be a deleting pendant $P_{2}$ operation, and deleting a pendant $P_{2}$ of $T$ or two pendant vertices of $T$ sharing a common neighbor is said to be a generalized deleting pendant $P_{2}$ operation.

For a tree on $n$ vertices, we can finally obtain $P_{1}$ for odd $n$ and $P_{2}$ for even $n$ by a series of generalized deleting pendant $P_{2}$ operations.

The following result has been obtained by Braga et al. [3]. Here we present a simple different reasoning.

Theorem 4.1. Let $T$ be a tree on $n \geq 2$ vertices. Then $m_{T}[0,2) \geq\left\lceil\frac{n}{2}\right\rceil$.

Proof. By Lemmas 2.3 and 2.4, each generalized deleting pendant $P_{2}$ operation decreases the number of Laplacian eigenvalues in $[0,2)$ by at least one. Thus, if $n$ is odd, then $m_{T}[0,2) \geq m_{P_{1}}[0,2)+\frac{n-1}{2}=\frac{n+1}{2}$, and if $n$ is even, then $m_{T}[0,2) \geq$ $m_{P_{2}}[0,2)+\frac{n-2}{2}=\frac{n}{2}$.

Lemma 4.1. Let $T$ be a tree with a diametrical path $P=v_{0} v_{1} \ldots v_{d}$, where $d \geq 4$, and for some $i$ with $2 \leq i \leq d-2, v_{i}$ is of degree three. Let $T^{\prime}=T-v_{i} v_{i+1}+v_{i}^{*} v_{i+1}$, where $v_{i}^{*}$ is the pendant neighbor of $v_{i}$ outside $P$. Then $m_{T}[0,2) \geq m_{T^{\prime}}[0,2)$.

Proof. Let us choose $v_{i}$ as the root of both $T$ and $T^{\prime}$. It is easily checked that $a_{T}(x)=a_{T^{\prime}}(x)$ for $x \in V(T) \backslash\left\{v_{i}, v_{i}^{*}\right\}, a_{T}\left(v_{i}^{*}\right)=-1$,

$$
\begin{gathered}
a_{T}\left(v_{i}\right)=2-\frac{1}{a_{T}\left(v_{i-1}\right)}-\frac{1}{a_{T}\left(v_{i+1}\right)}, \\
a_{T^{\prime}}\left(v_{i}^{*}\right)=-\frac{1}{a_{T^{\prime}}\left(v_{i+1}\right)}=-\frac{1}{a_{T}\left(v_{i+1}\right)}, \\
a_{T^{\prime}}\left(v_{i}\right)=-\frac{1}{a_{T^{\prime}}\left(v_{i-1}\right)}-\frac{1}{a_{T^{\prime}}\left(v_{i}^{*}\right)}=-\frac{1}{a_{T}\left(v_{i-1}\right)}+a_{T}\left(v_{i+1}\right) .
\end{gathered}
$$

Denote by $s$ the number of vertices in $T$ different from $v_{i}, v_{i}^{*}$ with negative weights. By Lemma 2.1, $m_{T}[0,2) \geq s+1$ and $m_{T^{\prime}}[0,2) \leq s+2$.

Suppose by contradiction that $m_{T}[0,2)<m_{T^{\prime}}[0,2)$. Then

$$
s+1 \leq m_{T}[0,2) \leq m_{T^{\prime}}[0,2)-1 \leq s+1,
$$

and thus $m_{T}[0,2)=s+1$ and $m_{T^{\prime}}[0,2)=s+2$, implying that $a_{T}\left(v_{i}\right) \geq 0, a_{T^{\prime}}\left(v_{i}^{*}\right)<$ 0 , and $a_{T^{\prime}}\left(v_{i}\right)<0$. From $a_{T^{\prime}}\left(v_{i}^{*}\right)<0$, we have $a_{T}\left(v_{i+1}\right)>0$, and then 


$$
a_{T^{\prime}}\left(v_{i}\right)-a_{T}\left(v_{i}\right)=a_{T}\left(v_{i+1}\right)+\frac{1}{a_{T}\left(v_{i+1}\right)}-2 \geq 0 .
$$

Thus $a_{T^{\prime}}\left(v_{i}\right) \geq a_{T}\left(v_{i}\right) \geq 0$, which is a contradiction.

Attaching the path $P_{2}$ to a vertex of a tree $T$ is called adding a pendant $P_{2}$ to $T$. By Lemma 2.3, each operation of adding a pendant $P_{2}$ increases the number of Laplacian eigenvalues in $[0,2)$ by one.

Theorem 4.2. Let $T$ be a tree on $n \geq 2$ vertices. Then $m_{T}[0,2)=\left\lceil\frac{n}{2}\right\rceil$ if and only if $\beta(T)=\left\lfloor\frac{n}{2}\right\rfloor$.

Proof. If $\beta(T)=\left\lfloor\frac{n}{2}\right\rfloor$, then by Theorem 3.2, we have

$$
\left\lceil\frac{n}{2}\right\rceil \leq m_{T}[0,2) \leq n-\left\lfloor\frac{n}{2}\right\rfloor=\left\lceil\frac{n}{2}\right\rceil,
$$

and thus $m_{T}[0,2)=\left\lceil\frac{n}{2}\right\rceil$.

Suppose that $m_{T}[0,2)=\left\lceil\frac{n}{2}\right\rceil$. We will prove that $\beta(T)=\left\lfloor\frac{n}{2}\right\rfloor$.

Claim 1. $T$ is a tree obtainable from $P_{2}$ if $n$ is even and from $P_{1}$ if $n$ is odd by sequentially adding pendant $P_{2}$ 's.

Applying a series of deleting pendant $P_{2}$ operations from $T$, we may finally obtain a tree $T^{(1)}$ without pendant $P_{2}$. Let $n^{(1)}=\left|V\left(T^{(1)}\right)\right|$. By Lemma 2.3, we have $m_{T^{(1)}}[0,2)=\left\lceil\frac{n^{(1)}}{2}\right\rceil$.

If $n^{(1)}=1$ or 2 , i.e., $T^{(1)} \cong P_{1}$ or $P_{2}$, then Claim 1 follows obviously. In the following, we will prove that $n^{(1)}=1$ or 2 .

Since $T^{(1)}$ has no pendant $P_{2}$, we have $n^{(1)} \neq 3$, and if $n^{(1)}=4,5$, then $T^{(1)}$ is a star, and thus $m_{T^{(1)}}[0,2)=n^{(1)}-1 \neq\left\lceil\frac{n^{(1)}}{2}\right\rceil$, which is a contradiction, implying that $n^{(1)} \neq 4,5$.

Suppose that $n^{(1)} \geq 6$. Let $d$ be the diameter of $T^{(1)}$, and let $P=v_{0} v_{1} \ldots v_{d}$ be a diametrical path of $T^{(1)}$. Note that both $v_{1}$ and $v_{d-1}$ are of degree at least three (since $T^{(1)}$ has no pendant $P_{2}$ ). If $d=2,3$, then $T^{(1)}$ is a double broom, by Theorem 3.3, $m_{T^{(1)}}[0,2) \geq n^{(1)}-2>\left\lceil\frac{n^{(1)}}{2}\right\rceil$, which is a contradiction. Thus $d \geq 4$.

Note that the deletion of edges in $P$ from $T^{(1)}$ results in a forest with $d+1$ components, each of which contains exactly one vertex of $P$. Among such $d+1$ components, denote by $T_{i}$ the one containing $v_{i}$, where $0 \leq i \leq d$.

Let $T^{(2)}$ be the tree obtained from $T^{(1)}$ by a series of generalized deleting pendant $P_{2}$ operations such that one vertex of $T_{i}$ is left if $\left|V\left(T_{i}\right)\right|$ is odd and two vertices of $T_{i}$ are left if $\left|V\left(T_{i}\right)\right|$ is even for all $2 \leq i \leq d-2$. Let $n^{(2)}=\left|V\left(T^{(2)}\right)\right|$.

Now by Lemmas 2.3, 2.4, and 4.1, we have 


$$
\begin{aligned}
\left\lceil\frac{n^{(1)}}{2}\right\rceil=m_{T^{(1)}}[0,2) & \geq m_{T^{(2)}}[0,2)+\frac{n^{(1)}-n^{(2)}}{2} \\
& \geq\left\lceil\frac{n^{(2)}}{2}\right\rceil+\frac{n^{(1)}-n^{(2)}}{2} \\
& =\left\lceil\frac{n^{(1)}}{2}\right\rceil .
\end{aligned}
$$

Thus $m_{T^{(2)}}[0,2)=\left\lceil\frac{n^{(2)}}{2}\right\rceil$.

Note that $P=v_{0} v_{1} \ldots v_{d}$ is still a diametrical path of $T^{(2)}, v_{1}$ and $v_{d-1}$ are both of degree at least three, and the vertices $v_{2}, v_{3}, \ldots, v_{d-2}$ are all of degrees two or three. This implies that the diameter, say $\bar{d}$, of $T^{(2)}$ satisfies that $4 \leq \bar{d} \leq n^{(2)}-3$.

If the vertices $v_{2}, v_{3}, \ldots, v_{d-2}$ in $T^{(2)}$ are all of degree two, then $T^{(2)}$ is a double broom, and by Lemma 2.2, we have

$$
\left\lceil\frac{n^{(2)}}{2}\right\rceil=m_{T^{(2)}}[0,2)=\left\lfloor\frac{2 n^{(2)}-\bar{d}}{2}\right\rfloor \geq\left\lfloor\frac{2 n^{(2)}-\left(n^{(2)}-3\right)}{2}\right\rfloor=\left\lfloor\frac{n^{(2)}+3}{2}\right\rfloor,
$$

which is a contradiction.

Suppose that there is a vertex $v_{i}$ of degree three in $T^{(2)}$, where $2 \leq i \leq d-2$. Denote by $v_{i}^{*}$ the pendant neighbor of $v_{i}$ in $T^{(2)}$ outside $P$. Let $T^{\prime}=T^{(2)}-v_{i} v_{i+1}+v_{i}^{*} v_{i+1}$. Note that $T^{\prime}$ has one less vertex of degree three than $T^{(2)}$. By Lemma 4.1, we have $m_{T^{(2)}}[0,2) \geq m_{T^{\prime}}[0,2)$. Repeating the transformation from $T^{(2)}$ to $T^{\prime}$, we can finally get a double broom $T^{*}$ with $n^{(2)}$ vertices such that the degrees of $v_{1}$ and $v_{d-1}$ in $T^{*}$ are the same as those in $T^{(2)}$, the vertices $v_{2}, v_{3}, \ldots, v_{d-2}$ and their pendant neighbors in $T^{(2)}$ are all of degree two in $T^{*}$, and $\left\lceil\frac{n^{(2)}}{2}\right\rceil=m_{T^{(2)}}[0,2) \geq m_{T^{*}}[0,2)$. Note that $T^{*}$ has diameter at most $n^{(2)}-3$ (since $v_{1}$ and $v_{d-1}$ are both of degree at least three). As above, we can deduce a contradiction.

Thus $n^{(1)}=1$ or 2 , and Claim 1 follows.

Obviously, each operation of adding a pendant $P_{2}$ increases the matching number by one. By Claim 1, $\beta(T)=\beta\left(P_{2}\right)+\frac{n-2}{2}=\frac{n}{2}$ if $n$ is even, and $\beta(T)=\beta\left(P_{1}\right)+\frac{n-1}{2}=$ $\frac{n-1}{2}$ if $n$ is odd. Thus $\beta(T)=\left\lfloor\frac{n}{2}\right\rfloor$.

\section{REMARK}

Recall that for a tree $T$ on $n \geq 2$ vertices, $\left\lceil\frac{n}{2}\right\rceil \leq m_{T}[0,2) \leq n-1$.

Theorem 5.1. For positive integers $n, k$ with $n \geq 2$ and $\left\lceil\frac{n}{2}\right\rceil \leq k \leq n-1$, there exists a tree $T$ on $n$ vertices such that $m_{T}[0,2)=k$. 
Proof. Observe that $m_{S_{2 k-n+2}}[0,2)=2 k-n+1$. Let $T$ be the $n$-vertex tree obtained by attaching a path on $2 n-2 k-2$ vertices to a vertex of $S_{2 k-n+2}$. By Lemma 2.3, we have

$$
m_{T}[0,2)=m_{S_{2 k-n+2}}[0,2)+\frac{2 n-2 k-2}{2}=k,
$$

as desired.

\section{ACKNOWLEDGMENTS}

This work was supported by the Specialized Research Fund for the Doctoral Program of Higher Education of China (No. 20124407110002) and the National Natural Science Foundation of China (No. 11071089).

\section{REFERENCES}

1. W. N. Anderson Jr. and T. D. Morley, Eigenvalues of the Laplacian of a graph, Linear Multilinear Algebra, 18 (1985), 141-145.

2. J. A. Bondy and U. S. R. Murty, Graph Theory with Applications, American Elsevier, New York, 1976.

3. R. O. Braga, V. M. Rodrigues and V. Trevisan, On the distribution of Laplacian eigenvalues of trees, Discrete Math., 313 (2013), 2382-2389.

4. M. Fiedler, Algebraic connectivity of graphs, Czechoslovak Math. J., 23 (1973), 298305 .

5. R. Grone and R. Merris, The Laplacian spectrum of a graph II, SIAM J. Discrete Math., 7 (1994), 221-229.

6. R. Grone, R. Merris and V. S. Sunder, The Laplacian spectrum of a graph, SIAM J. Matrix Anal. Appl., 11 (1990), 218-238.

7. J. Guo and S. Tan, A relation between the matching number and Laplacian spectrum of a graph, Linear Algebra Appl., 325 (2001), 71-74.

8. J. Guo, X. Wu, J. Zhang and K. Fang, On the distribution of Laplacian eigenvalues of a graph, Acta Math. Sin. (Engl. Ser.), 27 (2011), 2259-2268.

9. D. P. Jacobs and V. Trevisan, Locating the eigenvalues of trees, Linear Algebra Appl., 434 (2011), 81-88.

10. R. Merris, The number of eigenvalues greater than two in the Laplacian spectrum of a graph, Port. Math., 48 (1991), 345-349.

11. R. Merris, Laplacian matrices of graphs: A survey, Linear Algebra Appl., 197-198 (1994), 143-176. 


\author{
Lingling Zhou and Bo Zhou \\ Department of Mathematics \\ South China Normal University \\ Guangzhou 510631 \\ P. R. China \\ E-mail: zhoubo@scnu.edu.cn
}

\title{
Zhibin Du
}

School of Mathematics and Information Sciences

Zhaoqing University

Zhaoqing 526061

P. R. China 